\title{
Comparison of assessment scores for fatigue between multidimensional fatigue inventory (MFI-K) and modified chalder fatigue scale (mKCFQ)
}

\author{
Eun-Jin Lim $^{1}$ and Chang-Gue Son ${ }^{2^{*}}$ (1)
}

\begin{abstract}
Background: Because of the absence of biological parameters for fatigue, appropriate instruments for assessing the degree of fatigue are important in the diagnosis and management of people complaining of fatigue-like symptoms. This study statistically analyzed the fatigue scores from two typical questionnaire-based instruments: the Korean version of the Multidimensional Fatigue Inventory (MFI-K) and the modified Chalder Fatigue Scale (mKCFQ).

Methods: Seventy participants (males $n=40$, females $n=30$, median age 48 years old, range of 25-67) were grouped into three groups ('mild' $=20$, 'moderate' $=42$, and 'severe' $=8$ ) according to self-reported fatigue levels using a 7-point Likert scale. The similarities and differences between two instrument-derived scores were analyzed using correlations ( $r$ ) and multidimensional scaling (MDS).

Results: The total scores of the two assessments were significantly correlated ( $r=75 \%, p<0.001)$, as were the subscores ('Total Physical fatigue': $r=76 \%, p<0.001$,'Total Mental fatigue': $r=56 \%, p<0.001$ ). Relative overestimation of the MFI-K (45.8 \pm 11.3$)$ compared to the MKCFQ (36.1 \pm 16.2$)$ was observed, which was especially prominent in the 'mild' group. The scores of the three groups were more easily distinguished by the mKCFQ than by the MFI-K. In terms of the five dimension scores, we found a higher correlation of the two assessments for 'general fatigue' $(r=79 \%, p$ $<0.001)$ and 'physical fatigue' ( $r=66 \%, p<0.001)$ than for the reductions in 'motivation' $(r=41 \%, p<0.01)$ and 'activity' $^{\prime}(r=26 \%, p>0.05)$.

Conclusions: Our results may indicate the usefulness of the two instruments, especially for the physical symptoms of fatigue ('general' and 'physical' fatigue). Furthermore, the MFI-K may be useful for conditions of moderate-to-severe fatigue, such as chronic fatigue syndrome, but the mKCFQ may be useful for all spectra of fatigue, including in subhealthy people.
\end{abstract}

Keywords: Fatigue severity scale, Multidimensional fatigue inventory, Chalder fatigue scale, Chronic fatigue, Questionnaire

*Correspondence: ckson@dju.ac.kr

${ }^{2}$ Department of Korean Medicine, Institute of Bioscience and Integrative Medicine, Daejeon University, 62 Daehak-ro, Dong-gu, Daejeon 300-716, Republic of Korea

Full list of author information is available at the end of the article

\section{Background}

Fatigue is the state of weariness that may result from excessive physical and mental effort and psychological distress [1]. In general, fatigue is classified as acute, prolonged or chronic by the duration of the symptom, and chronic fatigue (fatigue $>6$ months) can be considered original author(s) and the source, provide a link to the Creative Commons licence, and indicate if changes were made. The images or other third party material in this article are included in the article's Creative Commons licence, unless indicated otherwise in a credit line to the material. If material is not included in the article's Creative Commons licence and your intended use is not permitted by statutory regulation or exceeds the permitted use, you will need to obtain permission directly from the copyright holder. To view a copy of this licence, visit http://creativecommons.org/licenses/by/4.0/. The Creative Commons Public Domain Dedication waiver (http://creativeco mmons.org/publicdomain/zero/1.0/) applies to the data made available in this article, unless otherwise stated in a credit line to the data. 
a medical problem [2]. Among types of chronic fatigue, chronic fatigue syndrome (CFS) is the most debilitating illness, characterized by post exertional malaise (PEM), sleep disorder, cognitive dysfunction, orthostatic intolerance and a seven-fold higher suicide rate [3]. Studies have found that up to $30-50 \%$ of the general population experienced fatigue $[4,5]$, approximately $10 \%$ experienced chronic fatigue [6], and a recent review study reported that $1 \%$ experienced CFS [7].

On the other hand, the recognition of fatigue has been expanded to various dimensions due to the complexity of fatigue $[2,8]$. Fatigue could be a physiological response as well as a disorder; however, there is no objective biological parameter to assess fatigue, which raises problems in the diagnosis and management of fatigue [9]. Accordingly, various fatigue measurement tools have been introduced to assess fatigability [2]. To date, diverse patient-reported outcome (PRO) measurements have been developed and used to assess fatigue status in clinics. Some are fatigue-nonspecific instruments, such as the 36-item Short Form Health Survey (SF-36) [10], Clinical Global Impression (CGI) [11], and Sickness Impact Profile-8 (SIP-8) [12], while fatigue-specific tools include the Checklist Individual Strength (CIS) scale [13], Chalder Fatigue Questionnaire (CFQ) [14], and Multidimensional Fatigue Inventory (MFI) [15].

We recently reviewed the trend of fatigue-assessment instrument application in clinical trials for CFS and found that both MFI and CFQ were the most commonly employed instruments [16]. These instruments reflect the clinical features of chronic fatigue divided into physical and mental domains and further into five dimensions: general, physical, mental, reduced activity, and reduced motivation $[14,15]$. In fact, several clinical studies have adopted MFI and CFQ as primary assessment tools for fatigue and CFS patients [17-21]. The selection of an appropriate assessment tool is crucial in fatigue-related clinical studies, while the Korean version of the MFI (MFI-K) and modified CFQ (mKCFQ) were designed for assessing the therapeutic process of Koreans complaining of fatigue and CFS, and each of them was clinically validated [22, 23]. However, no studies have been conducted comparing the characteristics of tools, particularly the most commonly used tools: MFI and CFQ.

The present study aims to evaluate the correlations and find the similarities and differences between the two instruments in identifying those with fatigue to determine their optimal usefulness.

\section{Methods \\ Participants}

This study comparing the MFI-K and mKCFQ was conducted from September to December 2020 among people working in a university. We collected email addresses from the university emailing group system shared only for the employees. We sent an invitation email to the 250 general employees including: educational personnel, researcher, and administrative people, and asked to participate for the survey if they consider themselves having some level of fatigue. In total 70were recruited and agreed to participate (Table 1). Prior to the survey, the level of fatigue (using a 7-point Likert scale: 1 indicates 'no fatigue or minimal' 7 indicates 'most severe fatigue or intolerable') was assessed for the purpose of grouping the participants. According to the fatigue level, the participants were grouped into three categories: 'mild' (fatigue level: 1-2), 'moderate' (3-5), and 'severe' (6-7). Participants who might experience discomfort, who refused to participate, who were pregnant or who had a condition that may influence the results were excluded from this study.

\section{Study instruments}

The MFI is an instrument with 20 questions (5-point Likert scale, $1=$ 'agree' to $5=$ 'disagree' for the positive and negative questions) assessing five dimensions of fatigue that are grouped into two parts: total physical fatigue (general, physical fatigue, reduced activity) and total mental fatigue (mental fatigue and reduced motivation). This instrument is designed with positive and negative questions to increase the reliability of the responses. In this study, we used the Korean version (MFI-K, Additional file 1: Table S1), which was validated with 595 participants experiencing fatigue in 2018 [22].

The CFQ is a PRO-based instrument composed of 11 questions ( 7 items for 'physical fatigue' and 4 items for 'mental fatigue') to assess the fatigue level based on a comparison with the "usual" status on a 4-point Likert

Table 1 Demographics of the participants

\begin{tabular}{|c|c|c|c|c|}
\hline \multirow[t]{2}{*}{ Participants } & \multicolumn{3}{|l|}{ Group $^{a}$} & \multirow[t]{2}{*}{ Total } \\
\hline & Mild & Moderate & Severe & \\
\hline Total, $n$ & 20 & 42 & 8 & 70 \\
\hline Median age (range) & $54(35-66)$ & $47(30-67)$ & $43(25-51)$ & $48(25-67)$ \\
\hline Mean BMI & $26.0 \pm 4.5$ & $25.6 \pm 4.8$ & $20.4 \pm 1.6$ & $25.2 \pm 5.2$ \\
\hline Male, $n$ & 15 & 22 & 3 & 40 \\
\hline Median age (range) & $56(35-66)$ & $48(32-64)$ & $44(42-50)$ & $50(32-66)$ \\
\hline Mean BMI & $31.0 \pm 4.1$ & $25.8 \pm 0.1$ & $26.0 \pm 1.2$ & $27.4 \pm 3.1$ \\
\hline Female, $n$ & 5 & 22 & 5 & 30 \\
\hline Median age (range) & $48(43-56)$ & $45(30-67)$ & $33(25-51)$ & $44(25-67)$ \\
\hline Mean BMI & $24.3 \pm 3.1$ & $25.5 \pm 5.3$ & $20.4 \pm 1.6$ & $23.9 \pm 4.3$ \\
\hline
\end{tabular}


scale ("Less than usual", "No more than usual", "More than usual", and "Much more than usual") [14]. The Korean version of the CFQ (K-CFQ) was validated with healthy participants in 2018 [24], and we used the modified Korean version (mKCFQ, Additional file 1: Table S1) in this study. To resolve the difficulty of assessment based on the comparison with the "usual" status, the mKCFQ was adapted to a 10-point Likert scale $(0=$ 'not at all' to $9=$ 'unbearably severe condition'), and its reliability and validity were confirmed with 97 CFS participants [23].

\section{Analysis of data}

For the consistent comparisons of the two instruments, the mKCFQ score (0-9 points for each of the 11 questions, maximum 99 points) was converted to a 100-point scale, the same as the MFI-K score (5 points each of the 20 questions, maximum 100 points). That is, the total score of each dimension of the mKCFQ (maximum 18 points for general, mental, reduced activity and motivation and 27 points for physical fatigue) was reformulated according to the MFI-K score, which was scored on a 20-point scale on each of the five dimensions (Additional file 1: Table S1). We compared and analyzed the data based on the five dimensions of fatigue.

The distributions and mean scores were determined and compared, and the correlations [Pearson $(r)]$ and $p$ values were assessed. The similarities and differences between the instruments were investigated regarding fatigue severity and the dimensions of fatigue. We also estimated the distance between the scores (standardized z-score) using multidimensional scaling (MDS) to visualize the similarities of the questions on the twodimensional space. MDS statistically assesses and clusters similar questions using the single-linkage method of hierarchical agglomerative clustering (HAC). MDS helps clarify the structure and relations between questions [25]. Kruskal's standardized residual sum of squares (STRESS) was also used to evaluate the goodness of fit of the instruments. The STRESS value ranges from 0 to 1 , and close to 0 is considered appropriate for the results [26]. The MDS ALSCAL method in SPSS v. 20 was used for the statistical analysis.

\section{Results}

\section{Characteristics of the participants}

A total of 70 participants (median age 48, range 25-67), including 40 males (median age 50, range 32-66) and 30 females (median age 44, range 25-67), were included. They were grouped into three groups according to fatigue level: 20 were 'mild', 42 were 'moderate', and 8 were 'severe.' The mean body mass index (BMI) was $25.2 \pm 5.2$. Age and BMI were higher in the 'mild' group than in the 'severe' group (age $p=0.002$, BMI $p=0.06$ ) (Table 1 ).

\section{Comparisons of the total scores of the MFI-K and mKCFQ according to fatigue level}

As expected, the total scores of both the MFI-K and mKCFQ showed a correlation with fatigue level (scored $1-7)$, while the correlation coefficients $(r)$ was higher for the mKCFQ $(r=0.76 \%, p<0.001)$ than for the MFI-K $(r$ $=0.57 \%, p<0.001)$. In addition, the mKCFQ score was more differentiated according to the fatigue level than the MFI-K score (mKCFQ: $\mathrm{R}^{2}=0.55$ vs. MFI-K: $\mathrm{R}^{2}=0.33$ ) (Fig. 1). Two instruments, the MFI-K and mKCFQ, also showed a good correlation, $r=75 \%(p<0.001)$ (Fig. 2).

\section{Comparisons of MFI-K and mKCFQ according to the five dimensions of fatigue}

The total MFI-K score $(45.8 \pm 11.3)$ was higher than the mKCFQ score $(36.1 \pm 16.2)$ for 70 participants. This pattern was found for both males (MFI-K 42.8 \pm 11.1 vs. mKCFQ $31.5 \pm 14.3)$ and females $(49.8 \pm 10.5$ vs. $42.2 \pm 16.8)$ and for both 'total physical fatigue' ( $28.1 \pm 7.3$ vs. $23.5 \pm 10.6)$ and 'total mental fatigue' (17.7 \pm 4.7 vs. $12.3 \pm 6.9)$. In comparisons of the correlation coefficient $(r)$ between the MFI-K and MKCFQ according to the five dimensions of fatigue, the 'total physical fatigue' score ( $r=76 \%, p<0.001)$ was highly correlated with the total 'mental fatigue score' $(r=56 \%, p<0.001)$. In particular, the scores of 'general' ( $r=79 \%)$ and 'physical' ( $r=66 \%)$ showed the highest correlations, while the lowest correlation was found for 'reduced activity' ( $r=26 \%$ ). This pattern was also seen when we separately compared male and female participants (Table 2).

\section{Comparisons of differentiating power of MFI-K and mKCFQ according to severity group}

In Fig. 3a-c, the linear correlations of MFI-K and $\mathrm{mKCFQ}$, according to fatigue level (Likert scale 1-7), was presented. However, the mKCFQ was likely to more distinctively differentiate the mild, moderate, and severe groups than the MFI-K. In detail, the difference in the total score between groups was larger for the mKCFQ (e.g., 'Mild'-'Moderate' =15.1, 'Moderate'-'Severe' $=22.2$ ) than for the MFI-K (e.g., 'Mild'-'Moderate' $=5.4$, and 'Moderate'-'Severe' $=13.3$ ). The score difference between the MFI-K and MKCFQ was larger in the group with a lower fatigue level ('Mild') (e.g., MFI-K 40.4-mKCFQ $22.8=17.6$ ) but smaller in the group with severe fatigue ('Severe') (e.g., 59.1-60.1=1.0). These patterns were similarly repeated for the scores of 'total physical fatigue' and 'total mental fatigue' (Table 3; Fig. $3 \mathrm{a}-\mathrm{c}$ ). Moreover, the strongest correlation among the five dimensions was found for the 'general' score in the 'severe group $(r=91 \%, p<0.01)$. 

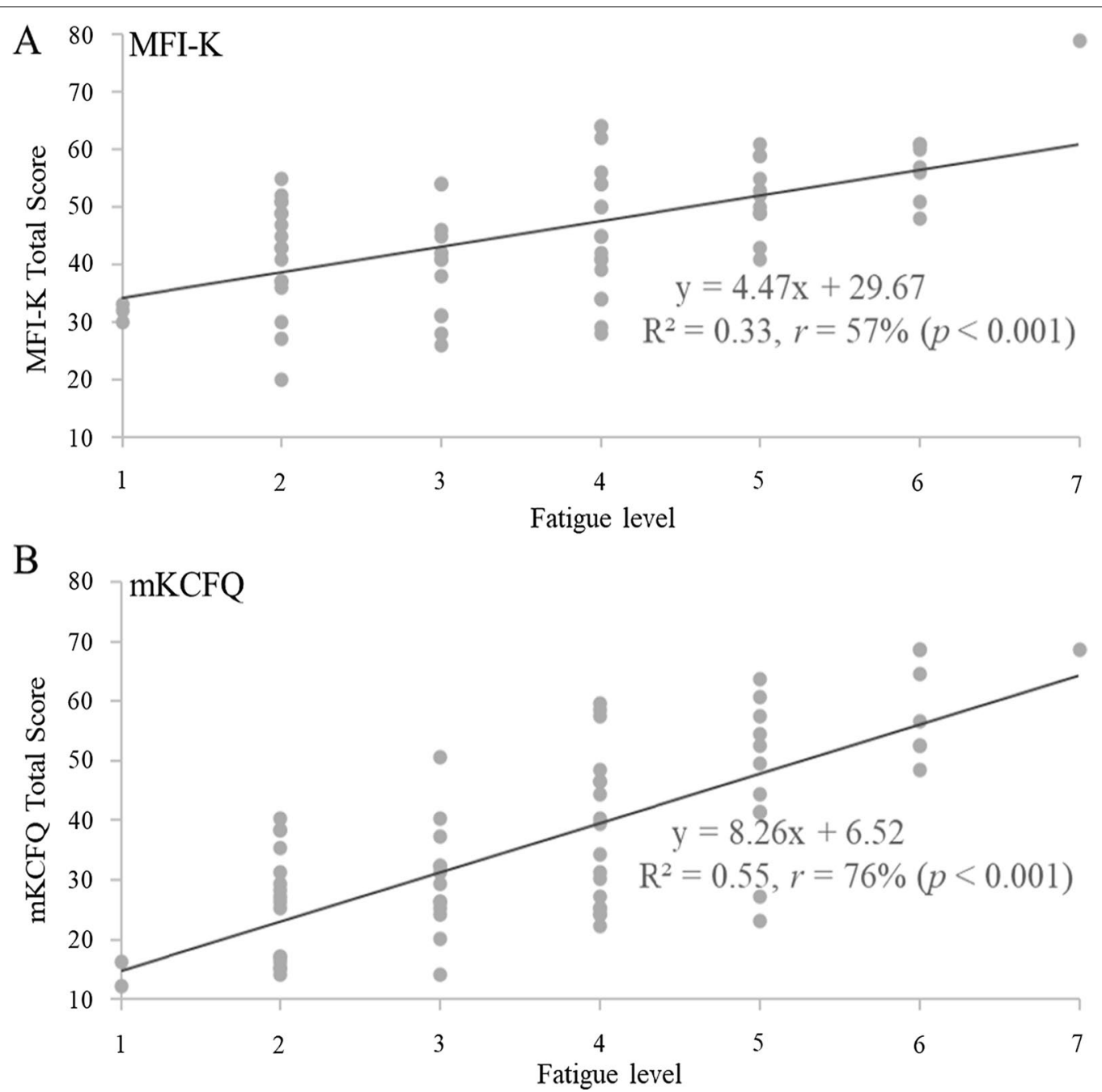

Fig. 1 Scatter chart of the total scores of the MFI-K and mKCFQ according to fatigue level

\section{Multidimensional scaling (MDS) analysis}

The distances of the scores between the MFI-K and mKCFQ were calculated using MDS analysis (Additional file 1: Table S2), and then they were structured on the two-dimensional Euclidean distance model (Fig. 4). The result clearly produced the separate components for the MFI-K and mKCFQ instruments (Dimension 1) and for 'physical' and 'mental' fatigue (Dimension 2), except the 'general' dimension of the MFI-K (M. General) and the 'reduced activity'(M. Activity) and 'motivation' (M. Motivation) dimensions of the MFI-K, which were conversely located far away from the other dimensions. The result of STRESS was 0.118 , which was considered to be fair and normal.

\section{Discussion}

The CFQ and MFI were initially developed to measure the severity of various fatigue types, including CFS, in England in 1993 [14] and to measure cancerrelated fatigue in the Netherlands in 1995 [15]. These two PRO-based instruments have been translated into as many languages and are commonly used for assessments of the extent and severity of fatigue in patient and nonpatient populations [27]. This study analyzed the similarities and differences of fatigue-assessment data from the Korean versions of MFI (MFI-K) and modified CFQ (mKCFQ) $[22,23]$ and aimed to produce important information regarding clinical choice and applications. 


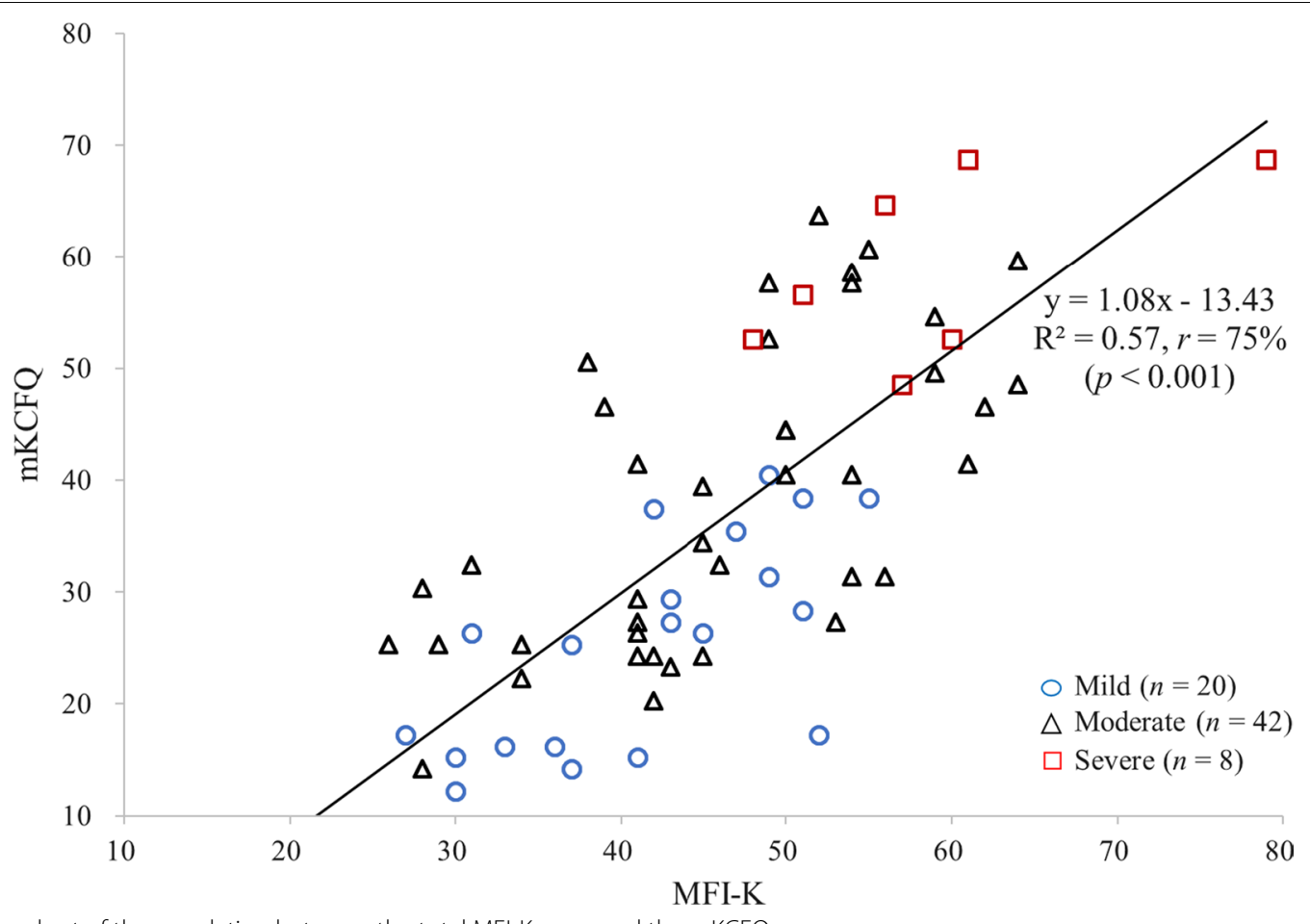

Fig. 2 Scatter chart of the correlation between the total MFI-K score and the mKCFQ score

Table 2 Correlation ( $r$ ) of MFI-K and mKCFQ in five dimensions $(n=70)$

\begin{tabular}{|c|c|c|c|c|c|c|c|c|c|}
\hline \multirow[t]{2}{*}{ Dimensions } & \multicolumn{3}{|c|}{ Total $(n=70)$} & \multicolumn{3}{|c|}{ Male $(n=40)$} & \multicolumn{3}{|c|}{ Female $(n=30)$} \\
\hline & MFI-K & mKCFQ & $r$ & MFI-K & mKCFQ & $r$ & MFI-K & mKCFQ & $r$ \\
\hline Total physical fatigue & $28.1 \pm 7.3$ & $23.5 \pm 10.6$ & $76^{* * *}$ & $26.5 \pm 7.1$ & $20.5 \pm 9.7$ & $73^{* * *}$ & $30.3 \pm 6.9$ & $26.6 \pm 10.6$ & $76^{* * *}$ \\
\hline General & $10.4 \pm 3.7$ & $8.9 \pm 4.3$ & $79^{* * *}$ & $9.6 \pm 3.8$ & $7.7 \pm 3.9$ & $76^{* * *}$ & $11.3 \pm 3.0$ & $10.6 \pm 4.2$ & $82^{* * *}$ \\
\hline Physical & $9.1 \pm 3.0$ & $7.8 \pm 3.4$ & $66^{* * *}$ & $8.4 \pm 2.5$ & $6.8 \pm 3.0$ & $56^{* * *}$ & $10.2 \pm 3.2$ & $9.1 \pm 3.4$ & $70^{* * *}$ \\
\hline Activity & $8.6 \pm 2.5$ & $6.8 \pm 4.1$ & 26 & $8.5 \pm 2.7$ & $6.1 \pm 4.1$ & 22 & $8.8 \pm 2.2$ & $7.8 \pm 3.9$ & 33 \\
\hline Total mental fatigue & $17.7 \pm 4.7$ & $12.3 \pm 6.9$ & $56^{* * *}$ & $16.3 \pm 4.5$ & $10.8 \pm 5.9$ & $56^{* * *}$ & $19.5 \pm 4.4$ & $14.6 \pm 8.1$ & $49^{* *}$ \\
\hline Mental & $8.7 \pm 2.6$ & $6.7 \pm 3.6$ & $56^{* * *}$ & $8.1 \pm 3.3$ & $6.1 \pm 3.2$ & $54^{* * *}$ & $9.7 \pm 2.4$ & $7.6 \pm 3.9$ & $53^{* *}$ \\
\hline Motivation & $8.9 \pm 2.6$ & $5.6 \pm 3.6$ & $41^{* *}$ & $8.3 \pm 2.6$ & $4.7 \pm 3.0$ & $36^{*}$ & $9.8 \pm 2.3$ & $6.7 \pm 3.9$ & $38^{*}$ \\
\hline Total & $45.8 \pm 11.3$ & $36.1 \pm 16.2$ & $75^{* * *}$ & $42.8 \pm 11.1$ & $31.5 \pm 14.3$ & $76^{* * *}$ & $49.8 \pm 10.5$ & $42.2 \pm 16.8$ & $70^{* * *}$ \\
\hline
\end{tabular}

$r$ Pearson correlation coefficient

${ }^{*} p<0.05,{ }^{* *} p<0.01,{ }^{* * *} p<0.001$

To evenly compare these instrument-derived fatigue scores, we converted mKCFQ (maximum 99 points) scores to 100 point-fitted scores to ensure that it was assessed on the same scale as the MFI-K score (maximum 100 points). As we expected, the total MFI-K and mKCFQ scores were significantly correlated $(r=75 \%, p$ $<0.001$, Table 2; Fig. 2), but they showed some differences regarding severity groups and dimensions of fatigue for a single group comprising 70 participants. The MFI-K score was significantly higher than the mKCFQ score by 1.3-fold for 70 participants $(45.8 \pm 11.3$ vs. $36.1 \pm 16.2$, $p<0.001$ ), 40 male and 30 female participants (Table 2). This high-score result in MFI-K mainly came from the score difference (17.6 points) for the 'mild'-fatigue group $(40.4 \pm 9.7$ vs. $22.8 \pm 10.7$, Table 3$)$, which is shown clearly in the Box-Whisker plot (Fig. 3a). These results may explain the possibility of a tendency of MFI-K to be scored high, especially for the general population with a very low level of fatigue. In fact, this pattern of the MFI-K tool was seen in its initial validation study, which 

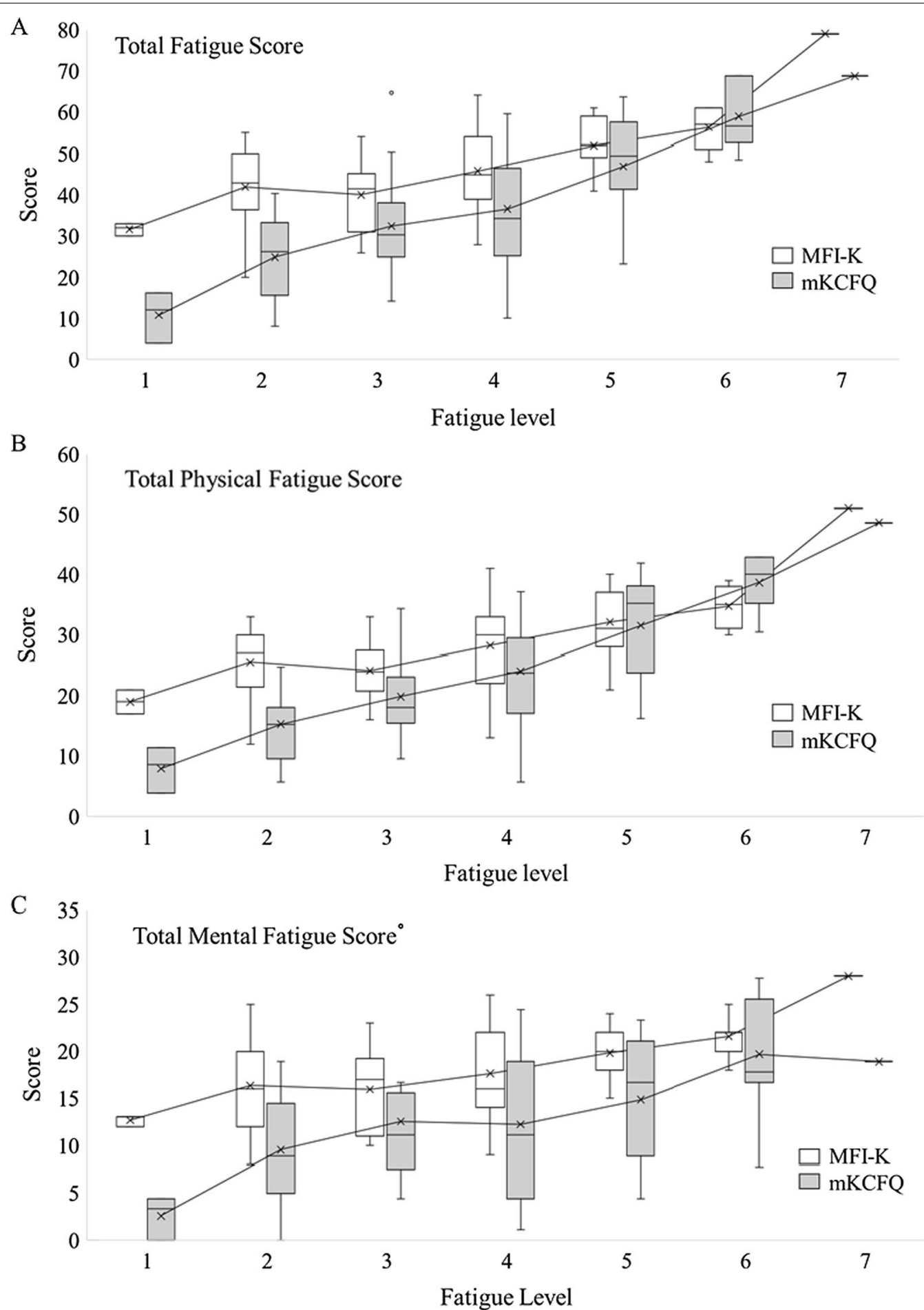

Fig. 3 Box-Whisker plot of the MFI-K and mKCFQ scores according to fatigue level. According to the fatigue level assessed on a 7-point Likert scale, the total fatigue score (A), total physical fatigue score (B) and total mental fatigue score $(\mathbf{C})$ of the MFI-K and mKCFQ are displayed

showed the comparison between the MFI-K total score (maximum 100) and VAS (maximum 100). In this study, compared to the 'severe' (5.5 points) and 'moderate' (19.9 points) groups, the 'mild' fatigue group ( 24.5 points) had the largest difference [22]. This can be due to MFI-K having more number of questions than $\mathrm{mKCFQ}$, thus MFI-K may be sensitive to certain aspects of fatigue questions. 
Table 3 Comparisons of mean scores ( \pm SD) by severity group

\begin{tabular}{|c|c|c|c|c|c|c|c|c|c|c|}
\hline \multirow[t]{2}{*}{ Group } & \multirow[t]{2}{*}{ Items } & \multicolumn{3}{|c|}{ Mild $(n=20)$} & \multicolumn{3}{|c|}{ Moderate $(n=42)$} & \multicolumn{3}{|c|}{ Severe $(n=8)$} \\
\hline & & MFI-K & mKCFQ & $r(\%)$ & MFI-K & mKCFQ & $r(\%)$ & MFI-K & mKCFQ & $r(\%)$ \\
\hline \multirow[t]{4}{*}{ Total physical fatigue (score 60) } & General & $7.3 \pm 2.5$ & $5.0 \pm 2.2$ & 32 & $10.9 \pm 2.8$ & $9.7 \pm 3.6$ & $66^{* * *}$ & $15.4 \pm 2.3$ & $14.7 \pm 2.6$ & $91^{* *}$ \\
\hline & Physical & $8.0 \pm 2.1$ & $5.6 \pm 2.7$ & 41 & $9.2 \pm 2.9$ & $8.0 \pm 3.0$ & $72^{* * *}$ & $11.9 \pm 3.8$ & $12.3 \pm 1.9$ & 25 \\
\hline & Activity & $9.4 \pm 2.5$ & $3.1 \pm 2.5$ & $65^{* *}$ & $8.1 \pm 2.5$ & $7.4 \pm 3.1$ & $44^{* *}$ & $9.5 \pm 2.1$ & $13.3 \pm 2.4$ & 19 \\
\hline & Total & $24.6 \pm 5.8$ & $14.2 \pm 6.1$ & $79^{* * *}$ & $28.1 \pm 6.8$ & $24.6 \pm 8.4$ & $69^{* * *}$ & $36.8 \pm 6.5$ & $39.9 \pm 5.5$ & 52 \\
\hline \multirow[t]{3}{*}{ Total mental fatigue (score 40) } & Mental & $7.4 \pm 2.5$ & $4.6 \pm 2.8$ & $57^{* *}$ & $9.0 \pm 2.4$ & $7.0 \pm 3.4$ & $43^{* *}$ & $11.1 \pm 2.0$ & $10.4 \pm 2.5$ & 21 \\
\hline & Motivation & $8.5 \pm 2.7$ & $3.9 \pm 2.9$ & 39 & $8.7 \pm 2.5$ & $5.7 \pm 3.3$ & $32^{*}$ & $11.3 \pm 1.4$ & $9.2 \pm 4.1$ & 40 \\
\hline & Total & $15.8 \pm 4.7$ & $8.5 \pm 5.6$ & $57^{* *}$ & $17.6 \pm 4.4$ & $12.7 \pm 6.4$ & $42^{* *}$ & $22.4 \pm 3.0$ & $19.6 \pm 6.3$ & 53 \\
\hline Total (score 100) & & $40.4 \pm 9.7$ & $22.8 \pm 10.7$ & $79^{* * *}$ & $45.8 \pm 10.4$ & $37.9 \pm 13.2$ & $65^{* * *}$ & $59.1 \pm 9.3$ & $60.1 \pm 8.5$ & 58 \\
\hline
\end{tabular}

The $r$ score and $p$ value are rounded to the 2 nd decimal

${ }^{*} p<0.05,{ }^{* *} p<0.01,{ }^{* * *} p<0.001$

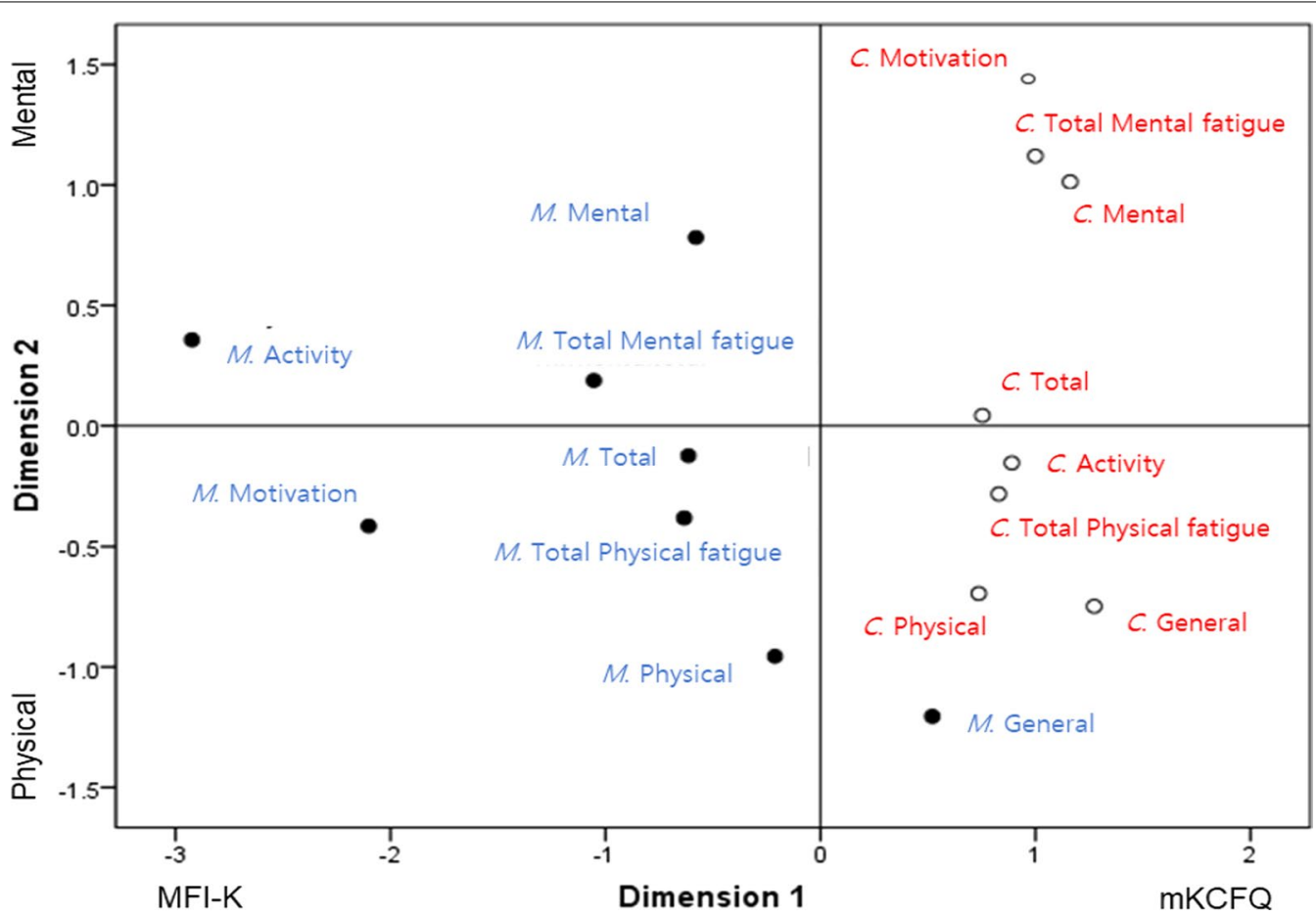

Fig. 4 Euclidean distance model (EDM) of multidimensional scaling (MDS). M MFI-K; C mKCFQ

In general, fatigue is grouped into two categories, socalled physical and mental fatigue, in most studies [28, 29]. The two instruments included in this study are also structured to distinguish between physical and mental fatigue assessments, and the responses to physical-mental fatigue-focusing questions were well differentiated from each other $[22,23,30]$. When we analyzed the correlation between the MFI-K and MKCFQ in the aspect of the physical and mental fatigue categories, each of the 'physical' and 'mental' fatigue scores correlated well between the two instruments, while the physical fatigue dimensions ('general', 'physical', and 'activity') were more highly correlated than the mental fatigue dimensions ('mental' and 'motivation', Table 2). We confirmed this feature in both box-whisker plots (Fig. 3b, c) and EDM analysis (Fig. 4). This would reflect the similarity of 'physical' fatigue assessments between two instruments but further dissimilarity of 'mental' fatigue assessments. In fact, previous studies using MFI reported similar results, showing a higher correlation of 'general' and 'physical' 
fatigue scores with VAS-based overall fatigue levels than scores of 'activity' and 'motivation' [15, 31]. These results may mean that the mKCFQ further sharply differentiates 'mental' fatigue severity from low-to-severe levels compared to the MFI-K.

Among the three fatigue groups and five dimensions, 'general' fatigue ( $r=91 \%$, MFI-K vs. $\mathrm{mKCFQ}=15.4 \pm 2.3$ vs. $14.7 \pm 2.6)$ and 'reduced activity' ( $r=19 \%$, MFI-K vs. $\mathrm{mKCFQ}=9.5 \pm 2.1$ vs. $13.3 \pm 2.4$ ) showed the highest and lowest correlations in the 'severe' fatigue group (Table 3). One possible reason might be the misunderstanding of questions such as "I feel very active", "I think I do a lot in a day", and "I feel like doing all sorts of nice things" in the MFI-K, which may seem less likely related to fatigue-related symptoms but rather more generalbehavioral questions (Additional file 1: Table S1). This is reflected in the two-dimensional matrix in Fig. 4, which, unlike the others, reduced 'activity' in the mental dimension (Fig. 4). From the results above, we suspect that the questions for reduced 'activity' and 'motivation' in MFI-K possibly misled the responses. In fact, a study of the reliability and validity of the MFI-K using outpatients of the Department of Family Medicine showed very low correlations with the VAS ('reduced activity' 0.087, and 'motivation' 0.159) [22]. The present study showed a relatively better ability of the mKCFQ to appropriately assess the severity of fatigue. In fact, CFQ has been criticized as an operational method that asks one to choose among "less than usual", "no more than usual", "more than usual", and "much more than usual", which may lead the answers to the extreme end of the scale [32]. Extreme scoring is likely to cause an inability to discriminate between different groups [32]. Thus, the mKCFQ was designed on a 10-point Likert scale, which allows for the assessment of small fatigue differences among participants and changes after therapeutic interventions [21,23].

In summary, the MFI-K and mKCFQ are likely to be sensitive in discriminating the 'severe' fatigue group, while the mKCFQ seems to be more suitable for participants with low levels of fatigue symptoms. In addition, clinicians or researchers may need to be aware of the low sensitivity of 'reduced activity' and 'motivation' relative to 'general' and 'physical' fatigue. For a certain illness such as CFS, the optimal fatigue scale should accurately identify specifications with multiple dimensions of fatigue in the process of both the diagnosis and assessment of therapeutics [33]. The MFI and CFQ and their Korean versions (MFI-K and mKCFQ) have been adapted in clinics and clinical trials for CFS patients [21, 23]; however, we still need to increase the specificity to differentiate patients by adding specific fatigue dimensions for postexertion malaise (PEM), one of the primary symptoms in patients with CFS [33]. This study has some limitations, such as a relatively small number of participants, especially for the 'severe' fatigue group and the inclusion of exclusively university personnel. This study was performed in Korea using Korean versions of fatigue scales; thus, limits in generalization with other languages. The correlations of the two instruments were compared based on the scores of the total and each domain. Further studies are needed among larger-scale populations with diverse fatigue severities.

\section{Conclusions}

We compared the Korean version of the MFI (MFI-K) and modified CFQ (mKCFQ) among a group of university personnel and analyzed the similarities and differences using correlations and the MDS. Overall, MFI-K and $\mathrm{mKCFQ}$ were highly correlated, while $\mathrm{mKCFQ}$ discriminated the severity of fatigue in a wider spectrum than MFI-K. Both instruments were more correlated for 'physical' symptoms than for reduced 'activity' and 'motivation. Further research is required to improve those dimensions to identify fatigue patients with multiple dimensions of fatigue, such as CFS.

\section{Abbreviations}

MFI-K: Korean version of multidimensional fatigue inventory; mKCFQ: Korean version of modified chalder fatigue scale; CFS: Chronic fatigue syndrome; MDS: Multidimensional scaling; EDM: Euclidean distance model.

\section{Supplementary Information}

The online version contains supplementary material available at https://doi. org/10.1186/s12967-021-03219-0.

Additional file 1: Table S1. Classification of the questions in sections for MFI-K and $\mathrm{mKCFQ}$. Table S2. Distance matrix.

\section{Acknowledgements}

This research was supported by the National Research Foundation of Korea (NRF) funded by the Oriental Medicine R\&D Project (NRF-2018R1A6A1A03025221).

\section{Authors' contributions}

L-EJ and C-GS conceptualization; L-EJ methodology, analysis and data collection, and writing; and C-GS review, editing, and supervision. All authors have read and approved the final manuscript.

\section{Availability of data and materials}

The data analyzed in this study are available from the first author on request.

\section{Declarations}

Ethics approval and consent to participate

The study was conducted in accordance with ethical and safety guidelines upon the approval of the Institutional Review Board of Daejeon University in South Korea (Approval number 1040647-201603-HR-001-01).

Consent for publication

Not applicable. 


\section{Competing interests}

The authors declare no competing interests.

\section{Author details}

${ }^{1}$ Department of Integrative Medicine, Graduate School of Integrative Medicine, CHA University, 335 Pangyo-ro, Sungnam-si, Bundang-gu 13488, Gyeonggi-do, Korea. ${ }^{2}$ Department of Korean Medicine, Institute of Bioscience and Integrative Medicine, Daejeon University, 62 Daehak-ro, Dong-gu, Daejeon 300-716, Republic of Korea.

Received: 26 July 2021 Accepted: 24 December 2021

Published online: 03 January 2022

\section{References}

1. Walters SJ, Stern C, Stephenson M. Fatigue and measurement of fatigue: a scoping review protocol. JBI Database SystRev Implement Rep. 2019;17:261-6.

2. Finsterer J, Mahjoub SZ. Fatigue in healthy and diseased individuals. Am J Hosp Palliat Care. 2013;31:562-75.

3. Institute of Medicine. Beyond myalgic encephalomyelitis/chronic fatigue syndrome: redefining an illness. 2015. https://www.nap.edu/catalog/ 19012/beyond-myalgic-encephalomyelitischronic-fatigue-syndromeredefining-an-illness. Accessed 20 Nov 2020.

4. Ricci JA, Chee E, Lorandeau AL, Berger J. Fatigue in the US workforce: prevalence and implications for lost productive work time. J Occup Environ Med. 2007:49:1-10.

5. van't Leven M, Zielhuis GA, van der Meer JW, Verbeek AL, Bleijenberg G. Fatigue and chronic fatigue syndrome-like complaints in the general population. Eur J Public Health. 2010;20:251-7.

6. Son CG. Review of the prevalence of chronic fatigue worldwide. J Korean Med. 2012;33:25-33.

7. Lim EJ, Ahn YC, Jang ES, Lee SW, Lee SH, Son CG. Systematic review and meta-analysis of the prevalence of chronic fatigue syndrome/myalgic encephalomyelitis (CFS/ME). J Transl Med. 2020;18:100.

8. Ericsson A. Fibromyalgia and chronic widespread pain Dimensions of fatigue and effects of physiotherapy. 2012. https://gupea.ub.gu.se/ handle/2077/28264. Accessed 5 Jan 2021.

9. Dukes JC, Chakan M, Mills A, Marcaurd M. Approach to fatigue: best practice. Med Clin N Am. 2021;105:137-48.

10. Stewart AL, Hays RD, Ware JE. The MOS Short-form general health survey. Med Care. 1988;26:724-35.

11. Guy W. ECDEU assessment manual for psychopharmacology. Washington: US Department of Health, Education, and Welfare; 1976.

12. Bergner M, Bobbitt RA, Carter WB, Gilson BS. The sickness impact profile: development and final revision of a health status measure. Med Care. 1981;19:787-805.

13. Vercoulen JHMM, Swanink CMA, Fennis JFM, Galama JMD, van der Meer JWM, Bleijenberg G. Dimensional assessment of chronic fatigue syndrome. J Psychosom Res. 1994;38:383-92.

14. Chalder T, Berelowitz G, Pawlikowska T, Watts L, Wessely S, Wright D, et al. Development of a fatigue scale. J Psychosom Res. 1993;37:147-53.

15. Smets EMA, Garssen B, Bonke B, De Haes JCJM. The multidimensional fatigue inventory (MFI) psychometric qualities of an instrument to assess fatigue. J Psychosom Res. 1995;39:315-25.

16. Kim DY, Lee JS, Son CG. Systematic review of primary outcome measurements for chronic fatigue syndrome/myalgic encephalomyelitis (CFS/ME) in randomized controlled trials. J Clin Med. 2020;9:3463.

17. Powell P. Randomised controlled trial of patient education to encourage graded exercise in chronic fatigue syndrome. BMJ. 2001;322:387-90.

18. Wearden AJ, Dowrick C, Chew-Graham C, Bentall RP, Morriss RK, Peters S, et al. Nurse led, home based self help treatment for patients in primary care with chronic fatigue syndrome: randomised controlled trial. BMJ. 2010;340:c1777.

19. Montoya JG, Kogelnik AM, Bhangoo M, Lunn MR, Flamand L, Merrihew $L E$, et al. Randomized clinical trial to evaluate the efficacy and safety of valganciclovir in a subset of patients with chronic fatigue syndrome. J Med Virol. 2013;85:2101-9.
20. Ostojic S, Stojanovic M, Drid P, Hoffman J, Sekulic D, Zenic N. Supplementation with guanidinoacetic acid in women with chronic fatigue syndrome. Nutrients. 2016;8:72.

21. Joung JY, Lee JS, Cho JH, Lee DS, Ahn YC, Son CG. The efficacy and safety of myelophil, an ethanol extract mixture of astragali radix and salviae radix, for chronic fatigue syndrome: a randomized clinical trial. Front Pharmacol. 2019;10:991.

22. Song SW, Kang SG, Kim KS, Kim MJ, Kim KM, Cho DY, et al. Reliability and validity of the Korean version of the multidimensional fatigue inventory (MFI-20): a multicenter, cross-sectional study. Pain Res Manag. 2018:2018:1-6.

23. Ahn YC, Lee JS, Son CG. Reliability and validity of the modified Korean version of the chalder fatigue scale (mKCFQ11). Healthcare. 2020;8:427.

24. Ha H, Jeong D, Hahm BJ, Shim EJ. Cross-cultural validation of the korean version of the chalder fatigue scale. Int J Behav Med. 2018;25:351-61.

25. Hout MC, Papesh MH, Goldinger SD. Multidimensional scaling. Wiley Interdiscip Rev Cogn Sci. 2013;4:93-103.

26. Kruskal JB. Multidimensional scaling by optimizing goodness of fit to a nonmetric hypothesis. Psychometrika. 1964;29:1-27.

27. Hewlett S, Dures E, Almeida C. Measures of fatigue: Bristol rheumatoid arthritis fatigue multi-dimensional questionnaire (BRAF MDQ), Bristol rheumatoid arthritis fatigue numerical rating scales (BRAF NRS) for severity, effect, and coping, chalder fatigue questionnaire (CFQ), checklist. Arthritis Care Res. 2011;63:S263-86.

28. Nozaki S, Tanaka M, Mizuno K, Ataka S, Mizuma H, Tahara T, et al. Mental and physical fatigue-related biochemical alterations. Nutrition. 2009;25:51-7.

29. Roelen CAM, Bültmann U, Groothoff J, van Rhenen W, Magerøy N, Moen $\mathrm{BE}$, et al. Physical and mental fatigue as predictors of sickness absence among Norwegian nurses. Res Nurs Health. 2013;36:453-65.

30. Cella M, Chalder T. Measuring fatigue in clinical and community settings. J Psychosom Res. 2010;69:17-22.

31. d'Elia HF, Rehnberg E, Kvist G, Ericsson A, Konttinen YT, Mannerkorpi K. Fatigue and blood pressure in primary Sjögren's syndrome. Scand J Rheumatol. 2008:37:284-92.

32. Stouten B. Identification of ambiguities in the 1994 chronic fatigue syndrome research case definition and recommendations for resolution. BMC Health Serv Res. 2005;5:37.

33. Jason L, Evans JM, Brown M, Porter N, Brown A, Hunnell J, et al. Fatigue scales and chronic fatigue syndrome: issues of sensitivity and specificity. Disabil Stud Q. 2011;31:1375.

\section{Publisher's Note}

Springer Nature remains neutral with regard to jurisdictional claims in published maps and institutional affiliations.

Ready to submit your research? Choose BMC and benefit from

- fast, convenient online submission

- thorough peer review by experienced researchers in your field

- rapid publication on acceptance

- support for research data, including large and complex data types

- gold Open Access which fosters wider collaboration and increased citations

- maximum visibility for your research: over $100 \mathrm{M}$ website views per year

At BMC, research is always in progress.

Learn more biomedcentral.com/submissions 